\title{
Theoretical and experimental Investigation of Structural, electronic and optical properties of neodymium doped $\mathrm{ZnO}$
}

\author{
Hajar Cherrad ${ }^{1 *}$, Mohammed Addou ${ }^{1,2}$, Mehdi Hssein ${ }^{1}$, Khadija Bahedi $^{1}$, Mohamed Jbilou ${ }^{2}$, Asmaa Mrigal $^{1}$, Elmehdi \\ Salmani ${ }^{4}$, Mustapha Rouchdi ${ }^{3}$, Ahmed Mezred ${ }^{3}$, Hajar Ftouhi ${ }^{2}$, Mustapha diani ${ }^{2}$, Mohamed El Jouad ${ }^{5}$ \\ ${ }^{1}$ Laboratoire Optoélectronique et Physico-chimie des Matériaux. Université Ibn Tofail, Faculté des Sciences BP 133 Kenitra 14000, \\ Morocco \\ ${ }^{2}$ Laboratoire de nanomatériaux et couches minces \\ Université Abdelmalek Essaadi- FST Tanger, Morocco \\ ${ }^{3}$ Materials Physics Laboratory, Mohammed V University, Faculty of Sciences, B.P. 1014, Rabat, Morocco \\ ${ }^{4}$ LMPHE, Faculté des Sciences, Université Mohammed V, Rabat, Morocco \\ ${ }^{5}$ Engineering Sciences Laboratory for Energy, National Engineering School of Applied Sciences. Chouaib Doukkali University of El \\ Jadida, Morocco.
}

\begin{abstract}
The morphological and cathodoluminescent properties of $\mathrm{Nd}$ doped zinc oxide thin films deposited by reactive chemical pulverization spray pyrolysis technique on heated glass substrates at 450 ${ }^{\circ} \mathrm{C}$ are being reported. The films were characterized by X-ray diffraction (XRD), scanning electron microscopy (SEM), and cathodoluminescent (CL) spectroscopy. We have also used the Generalised Gradient Approximation (GGA)-the Self Interaction Corrected (SIC) to determine Electronic structure and $\mathrm{x}$ ray absorption.
\end{abstract}

Keywords: thin film, neodymium, scanning electron microscope, cathodoluminescence, GGA-SIC.

\section{Introduction}

In recent years, zinc oxide has attracted much attention because of its novel optical and electrical properties. It is an n-type semiconductor with a wide direct band gap. The band gap is $3.44 \mathrm{eV}$ at low temperatures and 3.37 $\mathrm{eV}$ at room temperature [1].

These properties enable to have diverse applications in optoelectronics, especially in the blue/UV region, as well as potential applications in solar energy cells, organic light-emitting devices, electrostatic dissipative coatings, and chemical sensors [1-5].

Different techniques were used for thin films growth such as radio frequency (RF) magnetron sputtering [6], sol-gel [7], pulsed laser deposition [8], MOCVD [9], hydrothermal process [10] and spray pyrolysis technique [11]. The spray pyrolysis is a low cost technique, simple, and has the advantage of allowing deposition over large areas.

When $\mathrm{ZnO}$ is doped with rare earth ions, it allows both absorption of higher energy photons gap and a more efficient energy transfer to the rare earth ions. Different types of rares earths have been studied such as $\mathrm{Nd}, \mathrm{Yb}$ [12], Eu [13], Er [14], Ce [15], Tb [16] and Gd [17], which has emission peaks in the visible region. They can be used in many different applications, mainly scientific and industrial fields.

The objective of our work focuses on the preparation of thin films doped with neodymium, using the spray pyrolysis technique. The microstructure and morphology of the thin films have been studied by scanning electron microscopy (SEM), and a study of the cathodoluminescence of these thin films was performed. Lot of experimental and theoretical research results about Nd-doped has been published. Xian et al. [18] have investigated on the effect of $\mathrm{Nd}$ doping level on optical and structural properties of $\mathrm{Nd}$ thin films synthesized by the sol-gel route. The surface particles density is increased and all samples have UV emission centered at $377 \mathrm{~nm}$ and green emission centered at 511 $\mathrm{nm}$. The intensity of UV emission is greatly enhanced when $\mathrm{Nd}$ doping concentration is $1 \%$, and the green emission intensity is gradually increased with $\mathrm{Nd}$ doping concentration is increasing. Douayar et al. [19] have studied the investigation on the structural, optical and electrical properties of Nd-doped thin films deposited by spray pyrolysis. They have reported that the structure of the films is not sensitively modified by incorporation of $\mathrm{Nd}$.

The second part of this work focuses on theoretical studies using ab-initio method to determine electronic, optical properties of $\mathrm{Nd}$ doped. However, In order to model the extremely correlated $4 \mathrm{f}$-related electronic states, The self-interaction corrected "SIC" approach developed by Toyoda [20] within the KKR-CPA-SICGGA package as implemented into MACHIKANEYAMA2002 [21] is used for a more realistic description of the disordered local moments of the materials under investigation. The GGA-SIC approximation can be viewed as an extension of GGA, the Kohn-Sham wave function are projected onto a set of localized basis orbital's, the SIC approximation is governed by the energy difference between the energy

* Corresponding author: ch.hajar@gmail.com 
gain due to hybridization of the orbital with the valance band and the energy gain upon localization of the orbital [22]. This technique gives remarkable improvement of the electronic correlation of the transitions metals d states [23] and $\mathrm{Nd}$ f states [24].

\section{Experimental detail}

Nd-doped films were deposited by spray pyrolysis technique. Zinc chloride was dissolved in anhydrous ethanol to provide solution. The total concentration of metal ions was maintained at $0.05 \mathrm{~mol} / \mathrm{L}$. The $\mathrm{Nd}$ doping ratio defined by $[\mathrm{Nd}] /[\mathrm{Nd}+\mathrm{Zn}]$ has varied over a range of $1 \%, 3 \%$ and $5 \%$. The resulting mixture solution has been stirred for three hours at $50^{\circ} \mathrm{C}$ and agitated for one day at room temperature to yield a clear and transparent solution. The resulting solutions have been sprayed on the heated glass substrates. In our lab scale spray station, the substrates are fixed to a metal plate heated at the desired temperature of pyrolysis, using an electrically resistive wiring. During spraying, the substrates are moved underneath the spray nozzle, fixed in position to obtain homogeneous surfaces with suitable thicknesses. The deposition has been performed at different substrates temperature, $450{ }^{\circ} \mathrm{C}$ for $10 \mathrm{mn}$ as time deposition [25].

The structural characterizations are performed using $\mathrm{X}$ ray diffraction (XRD) with $\mathrm{Cu} \mathrm{K} \alpha$ radiation $(\lambda=1054 \AA$ ). The surface morphology has been examined by scanning electron microscopy (SEM).

The cathodoluminescence (CL) spectra which have been performed at room temperature using a LEO-GEMINI 982 scanning electron microscopy (SEM), have operated from 1 to $30 \mathrm{kV}$ and equipped with field emission gun. The CL system is associated with a spectrometer (Triax 190 from JOBIN YVON), which allows monochromatic $\mathrm{CL}$ imaging as well as acquisition of CL spectra on very localized spots of a sample.

\section{Experimental results}

The structural properties of the undoped and $\mathrm{Nd}$ doped grown at $450{ }^{\circ} \mathrm{C}$ with an atomic concentration of 1,3 and 5 at. $\%$ are analyzed by XRD patterns in figure 1 . In this figure, we can see that there is no additional peak corresponding to secondary phase which could be observed within the detection limit of the XRD technique. It can be found that all the films are polycrystalline with the hexagonal Wurtzite structure and have a preferred orientation with the $\mathrm{c}$ axis perpendicular to the substrates. The crystallinity has improved by high (002) peak intensity $\left(\mathrm{FWHM}=0.2^{\circ}\right)$.

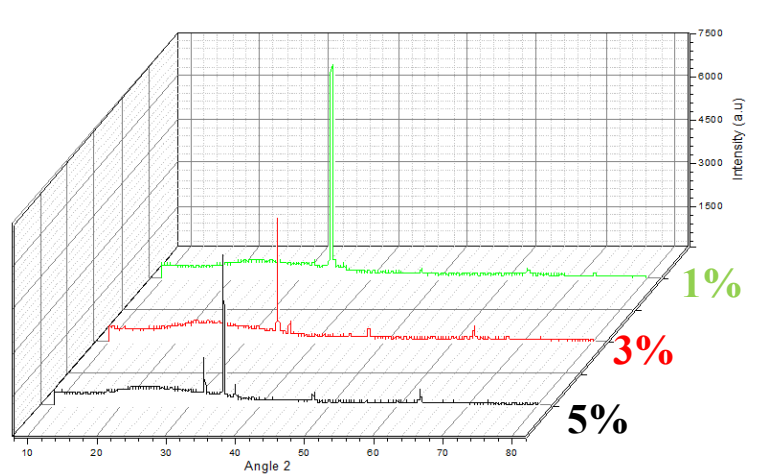

Fig. 1. XRD of doped $\mathrm{Nd}$ thin films

Fig. 2 shows the SEM micrographs of the undoped and 1 $\%, 3 \%$ and $5 \%$ Nd-doped thin films deposited at 450 ${ }^{\circ} \mathrm{C}$. The SEM photographs were taken at energy of $7 \mathrm{kV}$. We can see that all samples have dense grains. The grains are basically hexagonal, which helps the grains grow preferentially along the $\left[\begin{array}{lll}0 & 0 & 2\end{array}\right]$ crystallographic direction (c-axis) perpendicular to the substrates. This is due to the lowest surface energy of plane.

It can be seen that the surface morphology of the films strongly depends on the concentration of the dopant; we can notice that the grains size of $1 \% \mathrm{Nd}$ doped film is larger than that of the undoped film. The undoped film in Fig.2 represents a microstructure consisting of hexagonal-like grains of approximately $240 \mathrm{~nm}$ size. After the doping with $1 \%$, the size of the grains has increased. Besides, it has been observed that 3\% and $5 \%$ Nd-doped don't have uniform distribution. Comparing the undoped to the others samples, it has been observed that $1 \% \mathrm{Nd}$-doped thin film has uniform and dense grains, the size of the grains has increased to attend 320 $\mathrm{nm}$, which means that the incorporation of $1 \% \mathrm{Nd}$ has improved the crystalline property of films. These results matched well the morphology micrographs and XRD analysis, reported by Fenglin Xiann and Xiangyin Li [18].
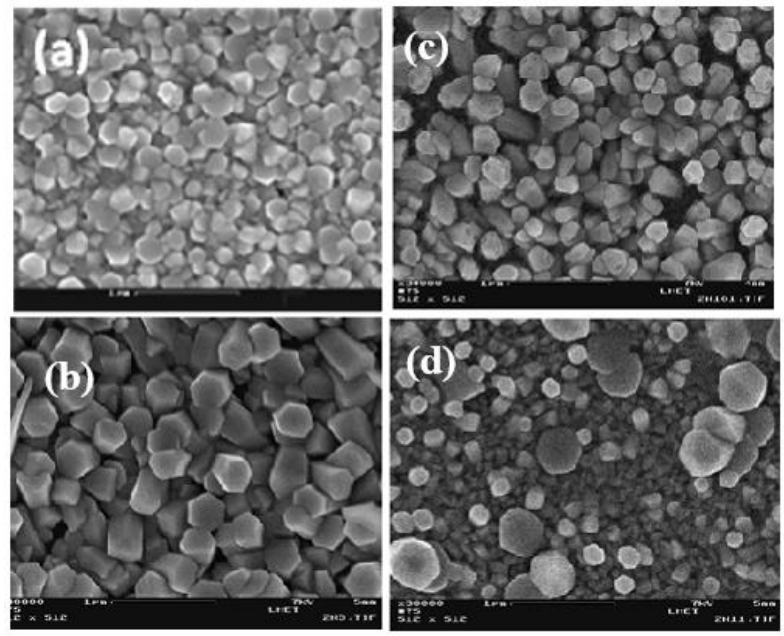

Fig. 2. SEM micrographs of: (a) undoped (b) 1 at.\% (c) 3 at. $\%$ and (d) 5 at.\% $\mathrm{Nd}$ doped thin films. 
Fig. 3 illustrates the emission spectra of the undoped and $\mathrm{Nd}$ doped samples. Early work has indicated that the spectra have exhibited three Cathodoluminescence bands centered on 380, 513 and $683 \mathrm{~nm}$, labeled: nearultraviolet (UV), blue-green and red bands [20]. The emission spectrum of our undoped sample consists of three peaks: 380,513 and $683 \mathrm{~nm}$. The $380 \mathrm{~nm}$ emission presents the UV peak and corresponds to the band gap transition of this material [26], which is also present in the Nd-doped spectrum. It is clear that the appearance of the band-edge corresponds to the good quality and crystallinity of our films. The second peak of the undoped sample presents the blue-green emission located at $513 \mathrm{~nm}$; this emission is discussed by Alaoui Lamrani and al. [26], but the origin of this emission is not clearly identified. They have attributed this transition to either gaps or excess oxygen and to radiative transitions between gaps of donor and acceptor oxygen deficiencies of zinc. The last emission at $683 \mathrm{~nm}$ shows low intensity comparing to the other emission and this is maybe due to the defect related to deep emission, as shown by photoluminescence [27]. Lohnert and al. [28] have pointed out that, the intensity of broad luminescence observed from about 450 to $700 \mathrm{~nm}$ depends on the oxygen partial pressure in the sintering process of ceramics. This also suggests the hypothesis of a transition within a Vox neutral and single-ionized oxygen $\mathrm{Vo}^{-}$.

The cathodoluminescence spectrum of Nd-doped films presents four emissions: high emission at $420 \mathrm{~nm}$ and three new emissions at $492 \mathrm{~nm}, 680 \mathrm{~nm}$ and $920 \mathrm{~nm}$. The UV emission depends on the density of free exciton in thin films; this emission results from recombination corresponding to the near edge emission.

Compared to the undoped, it can be seen that the intensity of ultraviolet emission is reduced when thin film is doped with $\mathrm{Nd}$. This is because the incorporation of small amount of $\mathrm{Nd} 3+$ ions in the lattice has been influenced by its crystallinity. In addition, we can see that $1 \% \mathrm{Nd}$ doped- has the biggest ratio of UV emission intensity, which confirms that $1 \% \mathrm{Nd}$ doped, effectively, reduces the defects in crystal, thus improve the crystalline quality.
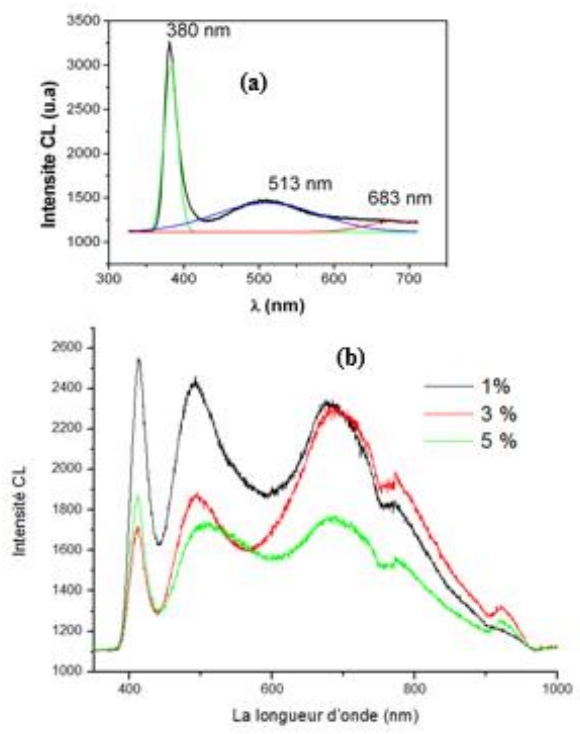

Fig. 3. Cathodoluminescence emission of undoped (a) and (b) Nd-doped films at different level doping.

The incorporation of neodymium in the lattice results in a competitive phenomenon that overshadows the bluegreen emission at $513 \mathrm{~nm}$ and generates new peak at 492 $\mathrm{nm}$, this visible emission is generally referred to deeplevel or trap-state emission, which is usually due to reduction of the self-activated centers responsible for the blue-green emission by neodymium ions occupation of zinc vacancies. The last luminescent band generated at $920 \mathrm{~nm}$ presents neodymium emission, this band originates from the $4 \mathrm{f}$ shell transition in the $\mathrm{Nd} 3+$ ions of lattice and corresponds to a transition from the excited states $(4 \mathrm{~F} 3 / 2)$ to the ground state $(4 \mathrm{I} / \mathrm{T} / 2)[29]$.

\section{Theoretical studies}

Figure 4 and 5 show the total and partial densities of state (DOS) and band structure of Nd calculated with GGA-SIC approximation. The $4 \mathrm{f}$ orbitals split into three parts due to the tetrahedral field: the lowest energy triply degenerates $\mathrm{t} 1 \mathrm{~g}$, triply degenerates $\mathrm{t} 2 \mathrm{~g}$ and singly degenerates with the highest energy state a $2 \mathrm{~g}$. All energies are relative to the respective Fermi level. Compared to the band structure of pure-, we note that the $\mathrm{Zn} 1-\mathrm{x} \mathrm{NdxO}$ with $\mathrm{x}=1,3$ and 5 calculated with GGASIC approximation is semi-conductive and has direct band gaps at $\Gamma$ point, similar to the wurzite structure given in Fig. 4. The Fermi energy is going into the conduction band after doping $\mathrm{Nd}$. The band gap decreases from $2.82 \mathrm{eV}$ of pure to $2.73 \mathrm{eV}, 2.70 \mathrm{eV}$ and $2.68 \mathrm{eV}$, for $\mathrm{Zn} 1-\mathrm{xNdxO}$ with $\mathrm{x}=1.3$ and 5 respectively, we note that the decrease in the gap is in agreement with experimental study with red shift of the excitonic peak observed in photoluminescence measurements for Nddoped $[30,31]$. 

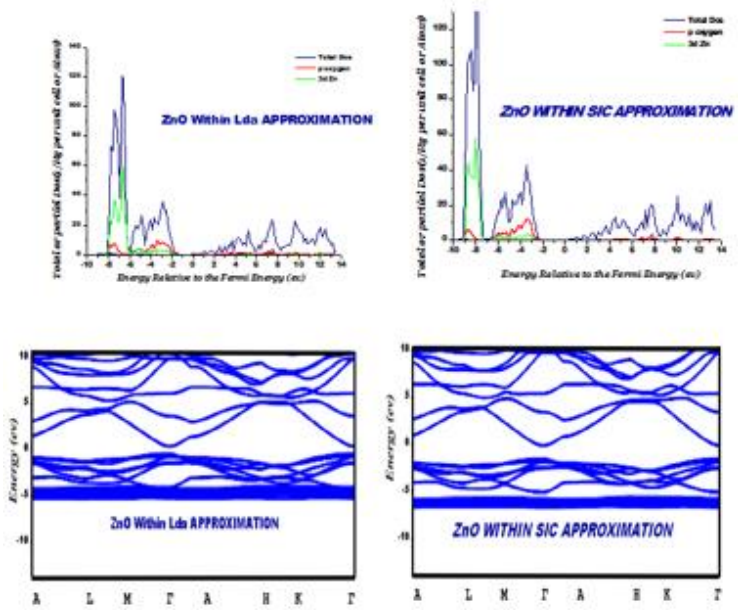

Fig.4. (a) (DOS) of $\mathrm{ZnO}$ and (b) Calculated band structure of $\mathrm{ZnO}$ calculated with GGA-SIC approximation.

X-ray absorption spectra play an important role in the optical properties of semiconductor materials. It is a very powerful investigation method of solid state physics which is used to study the structural, electronic and magnetic properties of materials. An x-ray photon can be absorbed by a core electron of an atom. The excited photoelectron moves to an empty state above the Fermi level. The probability of the transition depends on the energy of the absorbed photon. The absorption probability as a function of the photon energy ( $\mathrm{x}$ ray absorption spectrum) can be related to empty electronic states in the solid. Intense electronic transitions fulfil the dipole selection rules: the orbital and total moments of initial and final states change according to the relations $\Delta \mathrm{l}= \pm 1, \Delta \mathrm{j}=0, \pm 1$. Quadrupole transitions are less intensive and in this case the orbital and total moments of initial and final states are related to the quadrupole selection rules: $\Delta \mathrm{l}=0, \pm 2$ and $\Delta \mathrm{j}=$ $0, \pm 1, \pm 2$. If the excited core level has the principal number $\mathrm{n}=1$ the corresponding $\mathrm{x}$-ray absorption spectrum is called K-spectrum; if $\mathrm{n}=2$ then absorption spectrum is called L-spectrum [6].
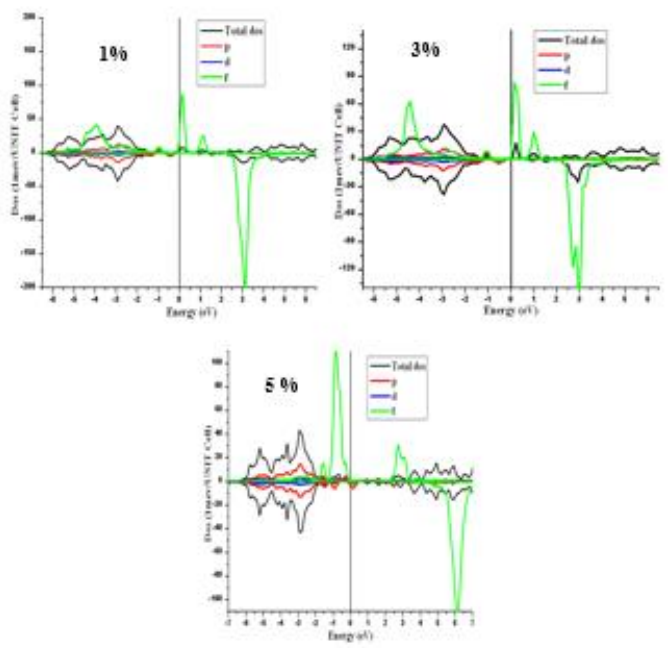

Fig. 5. total and partial densities of state (DOS) of : $\mathrm{ZnO}$ : $\mathrm{Nd}$.
The X-ray absorption spectra of $\mathrm{Zn}_{1-\mathrm{x}} \mathrm{Nd}_{\mathrm{x}} \mathrm{O}$ within GGA-SIC approximations are shown in Figure 6. It Corresponds to transitions of $1 \mathrm{~s}$ electrons of $\mathrm{TM}$ to empty states above the Fermi level. Although there are other possible transitions in this energy range (for example transitions of $2 \mathrm{~s}$ electrons), these transitions give only a very smooth contribution to the absorption spectrum while absorption caused by transitions of $1 \mathrm{~s}$ electrons changes very much near the absorption K-edge. This explains why the structure of the K-edge absorption spectra is determined by transitions of $1 \mathrm{~s}$ electrons.

Taking into account these transitions, we can calculate the absorption coefficient $\mu(\mathrm{E})$ using the dipole approximation:

$$
\mu(E) \infty\left|\left\langle\psi_{1 s}|e \nabla| \psi_{4 p}\right\rangle\right|^{2} \cdot n_{4 p}\left(\hbar \omega-E_{F}+E_{1 s}\right)
$$

When a $\mathrm{Nd}$ atom is in a substitutional position it is surrounded by four $\mathrm{Zn}$ atoms located on the vertices of a tetrahedron. The impurity states are determined by the interaction between dopant atomic states and the host states. The interaction of the atomic states with the valence band (VB) or conduction band (CB) would form bonding and antibonding states. The $4 \mathrm{f}$ orbitals split into three parts due to the tetrahedral field: the lowest energy triply degenerates $t 1 g$, triply degenerates $\mathrm{t} 2 \mathrm{~g}$, and singly degenerates with the highest energy state a $2 \mathrm{~g}$. After bonding $\mathrm{Nd}$ atom loses three electrons and becomes $\mathrm{Nd}^{3+}$.

X-ray absorption spectra of $\mathrm{Nd}$ doped within GGA approximations are shown in Figure 5. The shape of the spectra does not depend on the $\mathrm{Nd}$ content in $\mathrm{Zn}_{1-\mathrm{x}} \mathrm{Nd}_{\mathrm{x}} \mathrm{O}$.

Therefore the $\mathrm{Nd}$ atoms have the same valence and local crystal structure in all the samples. It is quite difficult to obtain more information from the spectra using such a simple reasoning. Ab-initio calculation methods will be used to determine the valence state of $\mathrm{Nd}$ in $\mathrm{Zn}_{1-\mathrm{x}}$ $\mathrm{Nd}_{\mathrm{x}} \mathrm{O}$. From the XANES spectra. However, only one pre-edge line is observed in the $\mathrm{K}$-edge $\mathrm{x}$-ray absorption spectra of $\mathrm{Nd}$ content in $\mathrm{Zn}_{1-\mathrm{x}} \mathrm{Nd}_{\mathrm{x}} \mathrm{O}$, the $2 \mathrm{p}$ states of $\mathrm{Nd}$ (spin up) are filled with valence state of $\mathrm{Nd}$ is $3+$.

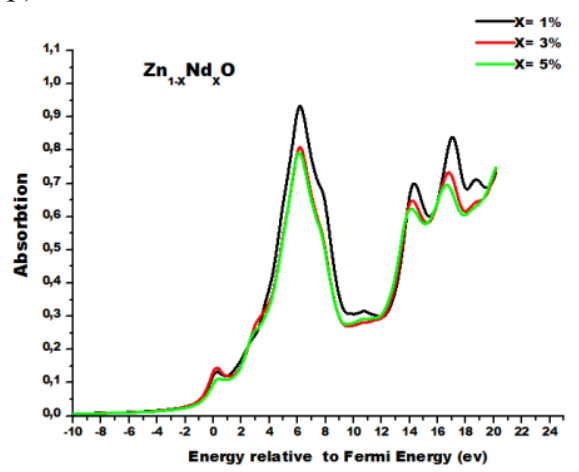

Fig. 6. X-ray absorption spectra of $\mathrm{Nd}$ doped $\mathrm{ZnO}$ within GGA. 


\section{Conclusion}

The morphological and optical properties of undoped and Nd-doped thin films prepared by the spray pyrolysis method have been reported in our experimental and theoretical studies. The surface morphology is investiged by scanning electron microscope. The results exhibit all samples that have hexagonal structure with a strong (002) preferred orientation. The Cathodoluminescence spectra measurements reveal that all samples have UV emission centered at $420 \mathrm{~nm}$, two blue green emissions centered at $492 \mathrm{~nm}$ and $680 \mathrm{~nm}$, infrared emission at 920 $\mathrm{nm}$. The Incorporation of neodymium in the lattice shifts the blue-green band to a blue at $492 \mathrm{~nm}$ and creates a new infrared emission at $880 \mathrm{~nm}$.

The theoretical study shows that the band gap decreases from $2.82 \mathrm{eV}$ of pure to $2.73 \mathrm{eV}, 2.70 \mathrm{eV}$ and $2.68 \mathrm{eV}$ for $\mathrm{Zn}_{1-\mathrm{x}} \mathrm{Nd}_{\mathrm{x}} \mathrm{O}$ with $\mathrm{x}=1,3$ and 5 respectively. The transitions give only a very smooth contribution to the absorption spectrum while absorption caused by transitions of $1 \mathrm{~s}$ electrons changes very much near the absorption K-edge. This explains why the structure of the K-edge absorption spectra is determined by transitions of $1 \mathrm{~s}$ electrons.

These films may be useful for specific applications for optoelectronic devices, such as light emitting diodes and solar cells transparent electrodes.

\section{References}

1. A. Janotti, C. G. Van de Walle, Fundamentals of zinc oxide as a semiconductor, Rep. Prog. Phys. 72 1-29 (2009).

2. Y. F. Hsu, Y. Y. Xi, C. T. Yip, A. B. Djurišić, W. K. Chan, Dyesensitized solar cells using tetrapods, Journal of Applied Physics. 103 1-4 (2008).

3. X. Jiang, F. L. Wong, M. K. Fung, S. T. Lee, Aluminum-doped zinc oxide films as transparent conductive electrode for organic light-emitting devices, Applied Physics Letters. 83 1876-1877 (2003).

4. M. Rydzek, M. Riedinger, M. Arduini-Schuster, J. Manara, Comparative study of sol-gel derived tin-doped indium and aluminum-doped zinc-oxide coatings for electrical conducting and low-emitting surfaces, Progress in Organic Coatings. 70 369-375 (2011).

5. H. Nanto, S. Tsubakino, T. Kawai, M. Ikeda, S. Kitagawa, M. Habara, Zinc oxide thin-film chemical sensors in conjunction with neural network pattern recognition for trimethylamine and dimethylamine gases, Journal of Materials Science. 29 65296532 (1994).

6. Y.C. Lin, M.Z.Chen, C.C. Kuo, W.T. Yen, Electrical and Optical Properties of $\mathrm{ZnO}$ :Al Film Prepared on Polyether sulfone Substrate by RF Magnetron Sputtering. Colloids and Surfaces A: Physicochem. Eng. Aspects. 337. (2009).

7. J.-P. Lin, J.-M. Wu, Scripta Mater. 60 (2009) 313-316.

8. Y. Liu, J. Lian, Optical and electrical properties of aluminum-doped $\mathrm{ZnO}$ thin films grown by pulsed laser deposition, Appl.Surf. Sci., Vol. 253, pp. 3727-3730, (2007).

9. M.E. Fragala and G. Malandrino, Characterization of $\mathrm{ZnO}$ and $\mathrm{ZnO}: \mathrm{Al}$ films deposited by MOCVD on oriented and amorphous substrates, Microelectronics
Journal, ,40(2), p.381-384 (2009).

10. J.T Chen, J. Wang, R.F. Zhuo, D. Yan, J.J. Feng, F. Zhang, P.X. Yan, The effect of Al doping on the morphology and optical property of $\mathrm{ZnO}$ nanostructures prepared by hydrothermal process. Appl. Surf. Sci.255 3959-3964 (2009).

11. A. Chakraborty, T. Mondal, S.K. Bera, S.K. Sen, R. Ghosh, G.K. Paul, Effects of Aluminum and Indium Incorporation on the Structural and Optical Properties of $\mathrm{ZnO}$ Thin Films Synthesized by Spray Pyrolysis Technique, Mater. Chem. Phys., 162-166 (2008).

12. N. Jiang, S. Ye, JR. Qiu. Electron energy-loss spectroscopy study of Yb doped. Journal of Applied Physics; 108(083535):14 (2010).

13. W. Badalawa, H. Matsui, T. Osone, N. Hasuike, H. Harima, H. Tabata. Correlation between structural and luminescent properties of Eu3p-doped epitaxial layers. Journal of applied physics; 109:053502-7 (2011).

14. Jang YR, Yoo KH, Ahn JS, Kim C, Park SM. 1.54 mm emission mechanism of Er-doped zinc oxide thin films. Applied Surface Science. 257:2822-4 (2011).

15. George A, Sharma SK, Chawla S, Malik MM, Qureshi MS. Detailed of X-ray diffraction and photoluminescence studies of Ce doped nanocrystals. Journal of Alloys and Compounds; 509:594-46 (2011).

16. Yang L, Tang Y, Hu A, Chen X, Liang K, Zhang L. Raman scattering and luminescence study on arrays of doped with Tb3p. Physical Review B: Condensed Matter (2008); 403:22304.

17. P.P. Murmu, J.V. Kennedy, A. Markwitz, B.J. Ruck. Compositional and structural study of Gd implanted films, 1151. Melville: American Institute of Physics;. 185-8 (2009).

18. F. Xiann, X. Li. Effect of $\mathrm{Nd}$ doping level on optical and structural properties of :Nd thin films synthesized by the sol-gel route. 509-511 (2012).

19. A. Douayar, P. Prieto, G. Schmerber, K. Nouneh, R. Diaz, I. Chaki, S. Colis, A. El Fakir, N. Hassanain, A. Belayachi, Z. Sekkat, A. Slaoui, A. Dinia and M. Abd-Lefdil, Investigation of the structural, optical and electrical properties of Nd-doped thin films deposited by spray pyrolysis. 1-3 (2013).

20. H. Akai: http://sham.phys.sci.osaka-u.ac.jp/ kkr/.

21. M. Toyoda, H. Akai, K. Sato, and H. Katayama-Yoshida, Electronic structures of (Zn, TM)O (TM: V, Cr, Mn, Fe, Co, and $\mathrm{Ni}$ ) in the self-interaction-corrected calculations. Physica B: Physics of Condensed Matter, Volume 376, p. 647-650 (2006).

22. J.P. Perdew, A. Zunger, Self-interaction correction to densityfunctional approximations for many-electron systems. Phys. Rev. B 235048 (1981).

23. G. K. H. Madsen and P. Novak, Charge order in magnetite. An LDA+U study. Europhys. Lett. 69, 777 (2005).

24. G. Caroena, W. V. M. Machado, J. F. Justo, L. V. C. Assali. Lanthanide impurities in wide bandgap semiconductors: a possible roadmap for spintronic devices. Appl. Phys. Lett. 102, 062101 (2013).

25. A. Mrigal, M. Addou, M. El Jouad, M. Hssein, and S. Khannyra. Electrochemical Stability and Large Optical Modulation the V2O5 Thin Films Made by Spray Pyrolysis. Sensor Letters Vol. 16, 1-7, (2018).

26. M. Alaoui Lamrani, M. Addou, Z. Sofiani, B. Sahraoui, J. Ebothe, A. El Hichou, N. Fellahi, J.C. Bernede, R. Dounia, Cathodoluminescent and nonlinear optical properties of undoped and erbium doped nanostructured films deposited by spray pyrolysis. 199 (2007).

27. A. El Hichou, M. Addou, J. Ebothe, M. Troyon, Influence of deposition temperature (Ts), air flow rate (f) and precursors on 
cathodoluminescence properties of thin films prepared by spray pyrolysis. 185 (2004).

28. K. Lohnert, E. Kubalek, Microscopy of Semiconducting Materials Institute Of physics Publishing, Bristol 303 (1983).

29. A. Karl, Jr. Gschneidner, and E. LeRoy, Handbook on the Physics and Chemistry of Rare Earths volume 4 Non-Metallic Compounds - II. 293 (1979).

30. M. Subramanian, P. Thakur, S. Gautam, K.H. Chae, M. Tanemura, T. Hihara, S. Vijayalakshmi, S.S. Kim, K. Asokan, $\mathrm{R}$. Jayavel, Intrinsic ferromagnetism and magnetic anisotropy in Gd-doped $\mathrm{ZnO}$ thin films synthesized by pulsed spray pyrolysis method.J . Phys. D: Appl. Phys. 42, 105410 (2009).

31. J.H. Zheng, J.L. Song, Z. Zhao, Q. Jiang, J.S. Lian, Optical and magnetic properties of $\mathrm{Nd}$ doped $\mathrm{ZnO}$ nanoparticles. Cryst. Res. Technol. 47, 713 (2012). 